\section{Is RARS-T a new disease entity or a subtype of RARS or ET?}

TO THE EDITOR: A recent report of "JAK2 V617F mutation in myelodysplastic syndrome, myelodysplastic syndrome/myeloproliferative neoplasm, unclassifiable, refractory anemia with ring sideroblasts with thrombocytosis, and acute myeloid leukemia" by Jekarl DW et al. [1] has shown one patient with JAK2 V617F mutation among 7 patients with RARS-T. This $14.3 \%$ of $J A K 2$ mutation rate seems to be lower than $58 \%$ of previously reported data in the review of Wardrop D and Steenma DP [2]. Fifty-four patients had JAK2 mutation among 93 patients with RARS-T in 10 serial studies. Additionally 2 of 27 JAK2 wild-type patients showed $M P L^{W 515}$ mutation. $M P L^{W 515}$ mutation is found in $5 \%$ of primary myelofibrosis and $1 \%$ of essential thrombocythemia (ET) cases [3]. But Jekarl DW et al did not report about this second mutation in their article. One more concern for this report was the incidence and the diagnosis of RARS-T. Seven patients were enrolled in this single-center study only for 3 years. Seven RARS-T patients seemed to be extraordinarily many, even when authors used WHO 2008 criteria which alleviates the criterion of thrombocytosis from $600,000 / \mu \mathrm{L}$ to $450,000 / \mu \mathrm{L}$, and their center is one of the large tertiary hospitals in Korea. Also, there was no data on 7 RARS-T patients about the percentages of ring sideroblast (RS), and comorbidities which are necessary for the exclusion of other reacitve thrombocytoses such as inflammation or bleeding.

The role of RS in the pathophysiology of RARS, RARS-T and RCMD-RS (this entity is recently combined with RCMD in WHO 2008 criteria) is not clearly elucidated now. Also the cut-off value of $15 \%$ is thought to be arbitrary.
Seventy-three MDS patients with RS showed the wide range of RS between $1 \%$ and $86 \%$ [4]. The survival of patients with RARS-T was similar to that of patients with ET but better than that of patients with RARS [5]. The thrombohemorrhagic complications of patients with RARS-T had not been reported except in some case repots. The recommended approach to RARS-T is same as in ET although anemia and neutropenia could be aggravated by cytoreductive therapy. Therefore, RARS-T, a provisional and new entity in WHO 2001 and 2008 criteria should be reconsidered whether it will be kept its own entity or be integrated into ET or RARS as a subtype depending on the dysplastic features of marrow.

Soo-Mee Bang, M.D.

Department of Internal Medicine, Seoul National University Bundang Hospital,

300, Gumi-dong, Bundang-gu, Seongnam 463-707, Korea Tel: +82-31-787-7039, E-mail: smbang7@snu.ac.kr

1. Jekarl DW, Han SB, Kim M, et al. JAK2 V617F mutation in myelodysplastic syndrome, myelodysplastic syndrome/myeloproliferative neoplasm, unclassifiable, refractory anemia with ring sideroblasts with thrombocytosis, and acute myeloid leukemia. Korean J Hematol 2010;45:46-50.

2. Wardrop D, Steensma DP. Is refractory anaemia with ring sideroblasts and thrombocytosis (RARS-T) a necessary or useful diagnostic category? $\mathrm{Br} \mathrm{J}$ Haematol 2009;144:809-17.

3. Pardanani AD, Levine RL, Lasho T, et al. MPL515 mutations in myeloproliferative and other myeloid disorders: a study of 1182 patients. Blood 2006;108:3472-6.

4. Juneja SK, Imbert M, Sigaux F, Jouault H, Sultan C. Prevalence and distribution of ringed sideroblasts in primary myelodysplastic syndromes. J Clin Pathol 1983;36:566-9.

This is an Open Access article distributed under the terms of the Creative Commons Attribution Non-Commercial License (http://creativecommons.org/licenses/by-nc/3.0) which permits unrestricted non-commercial use, distribution, and reproduction in any medium, provided the original work is properly cited. 
5. Schmitt-Graeff AH, Teo SS, Olschewski M, et al. JAK2 V617F mutation status identifies subtypes of refractory anaemia with ringed sideroblasts associated with marked thrombocytosis. Haematologica 2008;93:34-40.

THE AUTHORS' REPLY: Dr. Bang suggested that the incidence of $J A K 2$ V617F mutation in RARS-T was lower (14.3\%) than previously reported (58\%) and MPL mutation study was not performed. In case of RARS-T, the enrolled period of patients was from January 1999 to February 2010. We have found that the sentence written above was omitted and we are sorry for the mistake. As the number of patients was only 7, larger number of enrolled participant is required for the exact incidence as there were no data pertaining to Korean and Asian. Ethnic variation could be a possible explanation of the different incidence from previous publications. In this study, the method used for the detection of JAK2 V617F mutation was the real time PCR by melting curve analysis which has detection limit of less than $1 \%$. As the methods used in previous studies were various, there might have been differences in the detection limits of allele burden among the methods. However, because myelodysplastic syndrome (MDS) and myelodysplastic syndrome/myeloproliferative neoplasm (MDS/MPN) are hematopoietic stem cell diseases and the possibility of less than $1 \%$ of affected cells at the time of initial diagnosis is theoretically very low. The $M P L$ gene mutational study was performed in 4 of the RARS-T patients with available DNA and the results showed that none of them harbored $M P L$ exon 10 mutation. Additional studies for the associated genes are considered for further evaluation.

The diagnosis of RARS-T was defined according to the following WHO criteria [1]. The number of ring sideroblasts of 7 patients was from $21 \%$ to $68 \%$. As of comorbidities, one patient had diabetes mellitus with dilated cardiomyopathy and another patient had subclinical hypothyroidism. Because all patients had no written history of diseases causing thrombocytosis, the reactive thrombocytosis could be ruled out. None of the patients had bleeding or thrombotic complications.

We agree with the idea that RARS-T should be reconsidered whether it will be kept its own entity or be integrated into ET or RARS as a subtype depending on the dysplastic features of marrow. In our cases, only the case with $J A K 2$ V617F mutation showed platelet counts of more than $1,000 \times 10^{9} / \mathrm{L}$ and leukocytosis of $26.95 \times 10^{9} / \mathrm{L}$ with neutrophilia and the megakaryocytes showed atypical morphology of essential thrombocythemia (ET).

At least the RARS-T cases with the platelet count of greater than $1,000 \times 10^{9} / \mathrm{L}$ or $J A K 2$ V617F mutation should be included in ET rather than in RARS-T. Such cases could be classified as ET with ringed sideroblasts (ET-RS).

Dong Wook Jekarl, M.D. and Kyungja Han, M.D. Department of Laboratory Medicine, College of Medicine, The Catholic University of Korea

505, Banpo-dong, Seocho-gu, Seoul 137-701, Korea Tel: +82-2-2258-1644,E-mail: hankja@catholic.ac.kr

1. Swerdlow SH, Campo E, Harris NL, et al, eds. World Health Organization Classification of Tumors of Haematopoietic and Lymphoid Tissues. IARC Press Lyon 2008: 40-50.

DOI: $10.5045 / \mathrm{kjh} .2010 .45 .2 .140$ 